\title{
The Future of Fractional Lasers
}

\author{
Uwe Paasch, MD, $\mathrm{PhD}^{1}$ \\ ${ }^{1}$ Department for Dermatology, Venerology and Allergology, \\ University of Leipzig, Leipzig, Germany \\ Facial Plast Surg 2016;32:261-268.
}

\begin{abstract}
Address for correspondence Uwe Paasch, MD, PhD, Department of Dermatology, Venerology and Allergology, University of Leipzig, Philipp-Rosenthal-Strasse 23, 04103 Leipzig, Germany (e-mail: uwe.paasch@medizin.uni-leipzig.de).
\end{abstract}

\begin{abstract}
Keywords

- skin

- fractional

- laser

- drug delivery

- skin cancer
\end{abstract}

The human skin is one of the biggest organs in terms of surface. As humans grow older, more and more people are affected by skin diseases. Although a skin disease may be addressed easily due to its accessibility, the complex structure and function of it often prevent side effect-free interventions.

The observation that a fractionated treatment of the skin leads to improved results as compared with that which can be achieved by treating the whole affected area is not new. In vessels, it has been discovered that there is no need to address the structure in total along its way under the skin. In addition, there were superior cosmetic results when only parts of the vasculature were coagulated whereas intact structures did not diminish the results.

Fractional lasers are named after their characteristic to deliver a laser light onto fractions or parts of the skin surface. ${ }^{1}$ By doing so, it came to our attention that the biggest human organ in size is able to replace itself up to $50 \%$ within only 4 to 6 weeks if the individual piece of skin taken out is less than $0.3 \mathrm{~mm}$ in diameter. These columns of treated skin can go as deep as the entire integument and are called microscopic treatment zones (MTZ). As fractional lasers enable us to trigger a skin remodeling in both the epidermal and the dermal compartment, they have found a wide acceptance in a rather short time. On a cellular level, the post-fractional wound healing is defined by a stereotypical sequence of processes, mainly triggered by the activation of heat shock proteins (HSPs) within the remaining keratinocytes, culminating in an active expulsion of pigmented irreparable tissue parts (microscopic epidermal necrotic debris [MEND]) 3 days later. $^{2}$

Despite a wide use of fractional lasers to treat several skin conditions associated with aging and trauma, the door has just been opened in terms of using this extremely high capacity of self-renewal in the context of treating skin cancers, fibrosing skin diseases, inflammatory and granulomatous skin conditions, as well as infectious diseases. This review aims at summarizing the current status of fractional lasers in daily clinical routine as well as the underlying technology, and it provides an outlook for ways in which the as yet unused potential can be transferred to clinics.

\section{Basic Concepts and Mechanisms}

In the past it has been shown that wounding in particular results in a typical sequence of healing, which is most often accompanied by inflammation. To specifically address skin remodeling in sun-damaged or aged skin for a long time, socalled skin resurfacing has been used. Lasers that have been
Issue Theme Nonsurgical Facial Rejuvenation; Guest Editors, Tanja C. Fischer, MD, and Gerd G. Gauglitz, MD, MMS
DOI http://dx.doi.org/ 10.1055/s-0036-1584215. ISSN 0736-6825. 
used for this procedure were designed to remove the epidermal and parts of the dermal compartment and to apply heat. Although this procedure is still regarded as the gold standard in aesthetic medicine, its inborn adverse effects prevented it from widespread use. A breakthrough has been marked by the development of fractional lasers that work either in a nonablative or an ablative manner. ${ }^{1}$

Ensuring a treatment coverage of less than $50 \%$ of the skin surface, the skin uniformly heals by a unique spatiotemporal sequence. First within the remaining untreated epidermal compartment, HSPs are upregulated. The active expulsion mechanism called MEND is triggered. In parallel, cellular inflammation is triggered. The presence of $\mathrm{B}-, \mathrm{T}-$, and $\mathrm{CD}_{68}{ }^{+}$-cells is energy dependent and precedes the reformation of collagen and hyaluronic acid (HA) production. ${ }^{3}$ Later, it has been discovered that the concept of fractionated heating can also be achieved by application of radiofrequencies or microplasma.

Treating the skin in fractions has been made possible by both nonablative (NAFXL) and ablative (AFXL) fractional laser systems. To address the ablative effect in AFXL specifically, their MTZs are also called microscopic ablation zones (MAZ). Wavelengths of the mid-infrared spectrum $(1,300-1,550 \mathrm{~nm})$ are appropriate for NAFXL, whereas wavelengths of the farinfrared spectrum such as Er:YAG (2,940 nm), Er:YSGG (2,970 $\mathrm{nm})$, and $\mathrm{CO}_{2}$ laser $(10,600 \mathrm{~nm})$ are in use for AFXL. ${ }^{2}$ Recently, the Thulium laser $(1,920 \mathrm{~nm})$ has been added to the NAFXL portfolio. ${ }^{4}$

Despite the main type of tissue interaction determining wavelength, the coverage-that is, the percentage of treated skin-is the most important parameter to be chosen by the operator. The coverage itself is mainly determined by the spot size of the system which is mostly invariable and the density as expressed by MTZs or MAZ per square centimeter. The energy applied per MTZs or MAZ defines the depth of penetration and the coagulation zone as well as the corresponding heat delivery in relation to the pulse configuration. ${ }^{5}$ The overall shoot profile is further modulated by the type of scanner used, endogenous skin conditions (e.g., hydration), and other treatment modalities such as use of topical anesthetics and on-time cooling. ${ }^{2}$

Although NAFXL and AFXL systems use the same principle as a fractionated treatment, it is crucial to know that in case of ablation the epidermal barrier is disrupted up to $50 \%$ while AFXL systems are in use. However, this is not the case if MTZs are applied. Furthermore, even within the subgroup of ablative fractional lasers, the different wavelengths as well as many other laser parameters do influence the clinical endpoint of treatment. Therefore, it is crucial to have the appropriate training with a given machine to treat specific indications.

\section{Post-Fractional Wound Healing}

In response to a fractional laser treatment, focal overheating of epidermal as well as dermal structures results. Untreated epidermal skin parts respond with an upregulation of HSP (e.g., HSP70, HSP47) (- Fig. 2). Coagulation of the dermal compartment relates to the microscopically visible counterparts, the MTZs. In the case of AFXL, ablation zones are present. The diameters of ablation result from the spot sizes and the energy applied. The holes within the skin are surrounded by a coagulation zone in a three-dimensional manner. Their thickness mainly depends on wavelength, energy, and pulse configuration. However, the total thermal damage to the tissue is in nonlinear correlation to the energy applied. ${ }^{3}$

While an immediate response to the thermal stress and onto the dermal fibers is difficult to visualize, a strong upregulation of early-phase stress proteins over time within the untreated epidermal compartment is the first stereotypic step of post-fractional wound healing. An intact portion of approximately $50 \%$ of those cells ensures a scarless healing at the surface. During the following 24 to 48 hours, epidermal repair takes place. In other words, the time window of the fractionated opened epidermal barrier (TOR) is as long as 12 to 24 hours. This has many implications, from post-laser treatment care to the possibility of a facilitated delivery of substances, radiation, light, and any other material imaginable to the dermal compartment.

Along with the ongoing epidermal repair process, an inflammatory response becomes visible. Mixed cellular infiltrate is still visible histologically after 28 or more days if high energies are applied. Over time, collagen fibers, elastic fibers, and ground substance are replaced by newly synthesized molecules (-Fig. 1).

As a very unique and specific sign of post-fractional wound healing, the presence of so-called MEND becomes visible at the skin surface approximately 72 hours posttreatment. The MEND process reflects the removal of debris, melanin, elastin, and remnants of the coagulated dermal compartment and inflammatory cells. The process is considered to be an active expulsion process. Presumably, the quantity of MEND will be in relation to the extent of coagulated tissue remaining within the skin. However, in the case of very high densities and energies applied, the capacity of MEND may be too little and granuloma formation might occur.

\section{Evidence-Based Fractional Laser Treatments}

To date, fractional lasers are approved by the Food and Drug Administration (FDA) for a variety of indications such as rhytids, fine lines, skin texture, dyspigmentation, and vascular dyschromias. This has led to wide clinical use and is reflected by almost 1,000 publications. The study types among them range from randomized controlled studies to case reports and expert opinions. Having this body of evidence, reliable recommendations can be made based on the published evidence. ${ }^{6}$ The classes of evidence used ( - Table $\mathbf{1}$ ) have been derived from the distribution type of publications. Recommendations have been made to treat aged and sundamaged skin, wrinkles, scars, dyspigmentation, vascular conditions, and actinic keratosis.

Recently, fractional laser devices that can be used at home have been launched. Using low densities and fluences applied repetitively yield results while ensuring safety. 

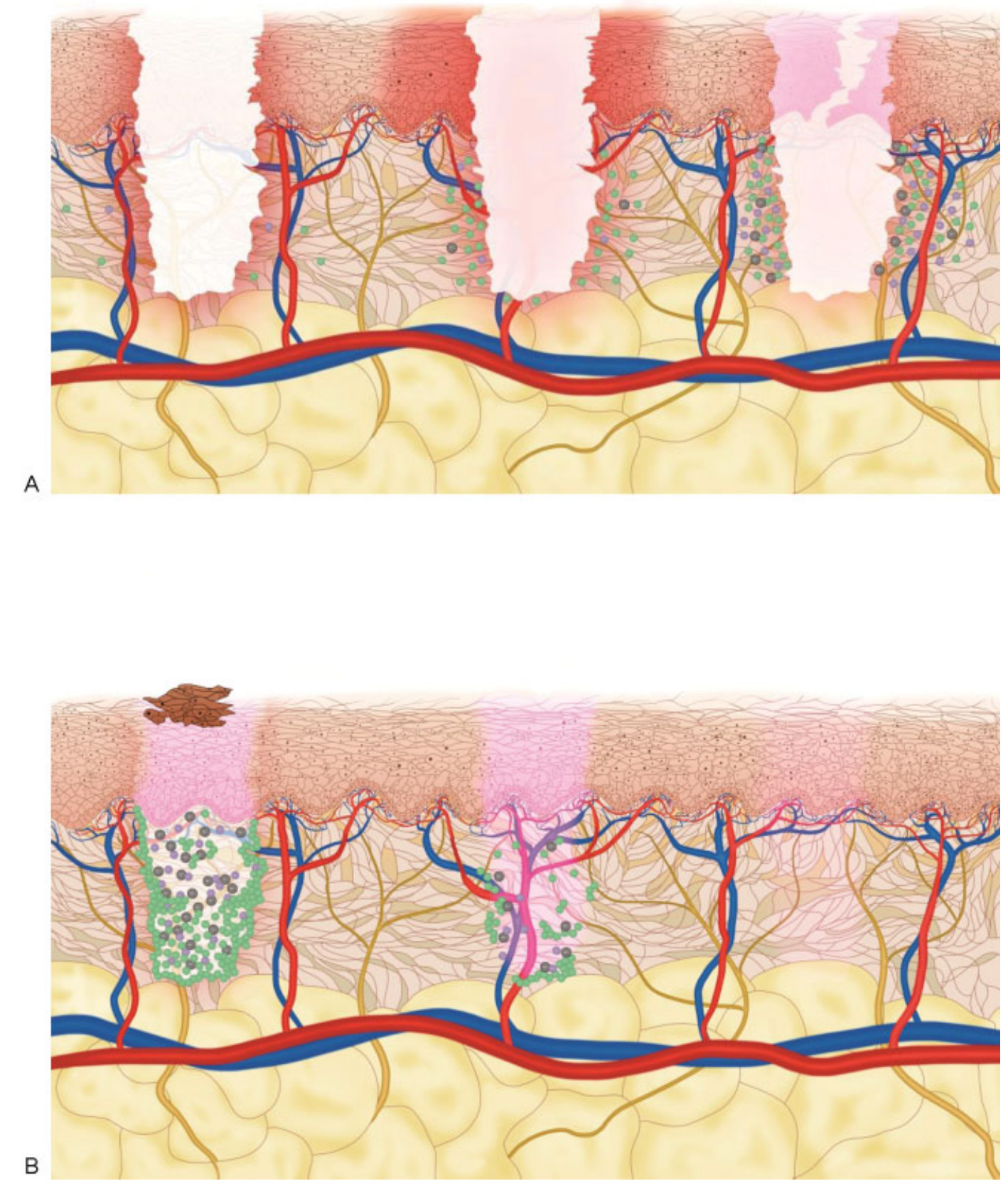

Fig. 1 (A, B) Sequence of post-ablative fractional laser system (AFXL) wound healing (from left to right). Reprinted with permission from Paasch et al. ${ }^{34}$

\section{Wrinkles and Ultraviolet-Damaged Skin}

The NAFXL procedures are well indicated to treat less severe wrinkled and ultraviolet (UV)-damaged skin. Deeper and dynamic wrinkles do need a more aggressive treatment using the AFXL technology. Ablative fractional $\mathrm{CO}_{2}$ lasers as well as Er:YAG lasers seem to achieve comparable results. However, in the end, the $\mathrm{CO}_{2}$ systems are regarded as much more efficient. ${ }^{6}$

\section{Scars}

Scars have been the main focus of interest when it comes to fractional lasers. Both the NAFXL and the AFXL systems have been shown to be highly effective on all skin types. In more than two-thirds of patients, an improvement has been seen. Although old scar tissue does respond, it came to our attention that treatment should be started as early as possible. With increasing treatment intensity, results seem to be 


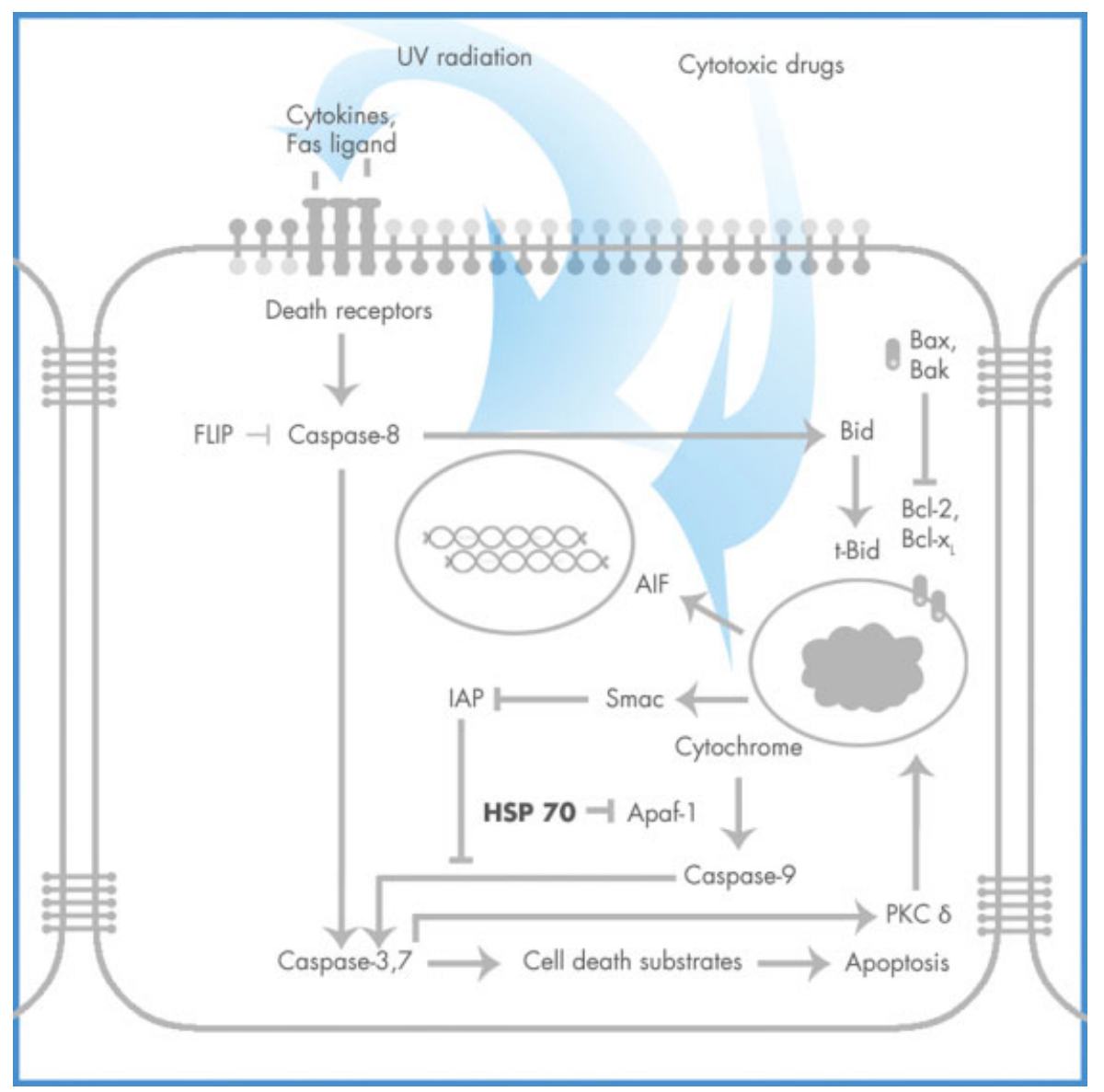

Fig. 2 Heat shock protein 70 (HSP-70) signaling pathway. AIF, anterior interbody fusion; Apaf-1, apoptotic protease activating factor 1; FLIP, FLICEinhibitory protein; IAP, inhibitor of apoptosis protein (IAP); PKC, protein kinase C delta type; UV, ultraviolet.

Table 1 Potential fields of ablative fractional laser (AFXL)-assisted drug delivery

\begin{tabular}{|l|l|}
\hline Skin rejuvenation & Hyaluronic acid, collagen stimulators \\
\hline Dynamic wrinkles & Argireline \\
\hline Scars and keloids & Matrix metalloproteinase inductors, mast cell stabilizers/inhibitors \\
\hline Striae & Not yet known \\
\hline Melasma & Melanosome inhibitors \\
\hline Tattoo & Inhibitors of phagocytosis \\
\hline Photodynamic therapy & Delta aminolevulinic acid (ALA), methyl-ester of ALA (MAL) \\
\hline Psoriasis & Vitamin-D3-analogs, methotrexate \\
\hline Vitiligo & 5-Phosphodiesterase inhibitors \\
\hline Acne & Retinoids \\
\hline Hair & 5-Dihydrotestosterone inhibitors/inducers, blue light \\
\hline Vessels & Yellow light \\
\hline Wounds & Growth factors \\
\hline Bacteria, fungi, Leishmania & Antibiotics, fungicides \\
\hline Granuloma anulare & Steroids, deep penetrating light \\
\hline
\end{tabular}

better; however, the risk of side effects escalates too. Less prominent scars do respond well to NAFXL, while thick scars and keloids have been reported to respond better to frequent $\mathrm{CO}_{2}$ laser treatments. However, recent improvements in
NAFXL systems that allow a deeper penetration might help favor the nonablative regimen in future. Interestingly, an ongoing inflammation has been reported not to influence the procedure negatively. ${ }^{6}$ 


\section{Dyspigmentation and Melasma}

As a matter of fact, the MEND shuttle is characterized by transporting melanin to the epidermal surface. This has created hope to treat dyspigmentations of the skin as well as the specific condition of melasma more effectively. On a cellular level, pigments stored in melanophages will be distributed within the tissue once cells have been disrupted by laser energy. As a consequence, the numbers of melanocytes and the amount of melanin stored in keratinocytes are reduced. Most often, this effect is transient only. In approximately $60 \%$ of the patients, improvement is seen in 75 to $100 \%$, although $30 \%$ reported an improvement of $25 \%$ only. Low densities and fluences are crucial, especially in darker skin types. It seems that the NAFXL gives more reliable results; however, it is not rated as a first-line intervention. ${ }^{6}$

\section{Vascular Disorders}

Since its introduction, the efficacy of NAFXL to treat hemangioma, postinflammatory erythema, and telangiectasia has been reported despite the fact that highly selective lasers are at hand. Looking at histology, it has been nicely shown that there is a wide intravascular coagulation due to NAFXL. ${ }^{6}$ The AFXL system seems to be beneficial for atrophic lesion or for those with scar tissue even in children. ${ }^{7}$ Hence, there is no specific absorber other than water, and lymphangioma can be also treated. ${ }^{8}$

\section{Actinic Keratosis and Skin Cancer}

A new and very promising treatment modality of fractional lasers has been established in the field of dermatological oncology. The first FDA approval of a 1,927-nm Thulium laser was based on a 83.5\% reduction of actinic keratoses (AK) at the face, arms, hands, and on the trunk. ${ }^{6}$ Comparable healing rates $(86.6 \%)$ confirmed this finding during a follow-up of 6 months. ${ }^{9}$ With regard to the evidence of other interventions to treat AK (e.g., photodynamic therapy [PDT], topical, cryotherapy), fractional laser therapy cannot be recommended as first-line intervention. ${ }^{6}$

The non-melanocytic neoplasms that are frequently associated with chronic actinic skin damage have led to the observation that the use of ablative fractional lasers results in enhanced healing compared with classical ablation of such lesions. However, AFXL alone is inferior to AFXL-assisted PDT. ${ }^{10}$

\section{Potential Future Use of Fractional Lasers}

MENDs and TORs Are Unique Features for Future Use

Two unique processes do highlight the post-fractional healing process. The scarless healing of approximately half of the organ by a complete tissue replacement due to an active renewal is outstanding. As there is a disease-related counterpart in the skin (reactive perforating dermatoses), the question arises if it is restricted to the skin or if the concept could be addressed to other epithelia such as mucosal membranes (leukoplakia) and to solid organs such as the liver in case of its fibrosis. As the MENDs seem to be actively transported, it would be interesting to know if it could be used for other purposes such as removal of foreign bodies (tattoo ink, dirt from traumata, parasites), granuloma, amyloids, and even localized infectious agents. To some extent, the concept has been proven in cases of tattoo allergy. ${ }^{11}$ Probably the most true capacity of this method of rejuvenation might be understood by the fact that in a progeria mouse model, aging of any organ could be reversed simply by clearance of p16Ink4apositive senescent cells. ${ }^{12}$

In fact, the first new route of transcutaneous drug delivery has been made possible for PDT. ${ }^{13}$ However, despite the experimental application of delta aminolevulinic acid, imiquimod, hydrochinon, dextrans, and melanocytes in vitro, there is still not much progress in vivo at this point. ${ }^{14-16}$ This may be attributed to the fact that many factors may influence the penetration capacity. ${ }^{17}$ First of all, there are dimensional problems next to the fluids and their pressures, and the fact that coagulation of dermal tissue may counteract in that process.

To date there are data from in vitro studies available showing that through the TOR, photosensitizers can be brought very deep into the skin and can be distributed more evenly. ${ }^{14}$ As a clinical consequence, the combination of AFXL and PDT has already been proven to be superior in the treatment of field cancerization. ${ }^{18}$ As it came to our attention that this specific scarless skin opening has been made possible, it has been the object of speculation. The potential for use with drug delivery is obvious ( $\mathbf{- T a b l e ~} \mathbf{1}$ ).

Further research is ongoing to understand how larger molecules or highly viscous substances can be put through the TOR in a safe and effective manner. Technologies used to overcome limited permeation are sonophoresis, iontophoresis, electroporation, or hydroporation using external forces.

First in vitro investigations have proved that HA can be deposited with the deep dermal tissue (-Figs. 3). As HA is optically not dense, an immediate filling of MAZ with HA by extracorporal forces using sonophoresis would establish an optical transparent window to the dermis. Additionally, it not only supports the natural wound healing but may also serve as a carrier for other active substances such as photosensitizers or nanovehicles. Nanocarriers are an emerging platform not only for cancer treatment. ${ }^{19}$ Owing to the ideal size relationships of ablation zones and the miniaturization of those carriers, ideal combination appears. So far, carbon tubes, fusion proteins, liposomes, capsosomes, micelles, dendrimers, nanoshells, polymeric carriers next to larger antibodies, PEGs, and dextrans are at hand $\left(-\right.$ Fig. 4). ${ }^{20}$

Even more fascinating is the idea that through those transparent columns of HA within the dermal compartment, it might be exposed to light of wavelengths and intensities which otherwise would never reach this area. There are also many implications. Within the current content, the use of UV and blue light might help enhance the efficacy of PDT in deep dermal structures, and melanocytes of hair follicles may be stimulated and presumably contribute to hair re-colorization. Vascular lesion may be much more effectively targetable and 


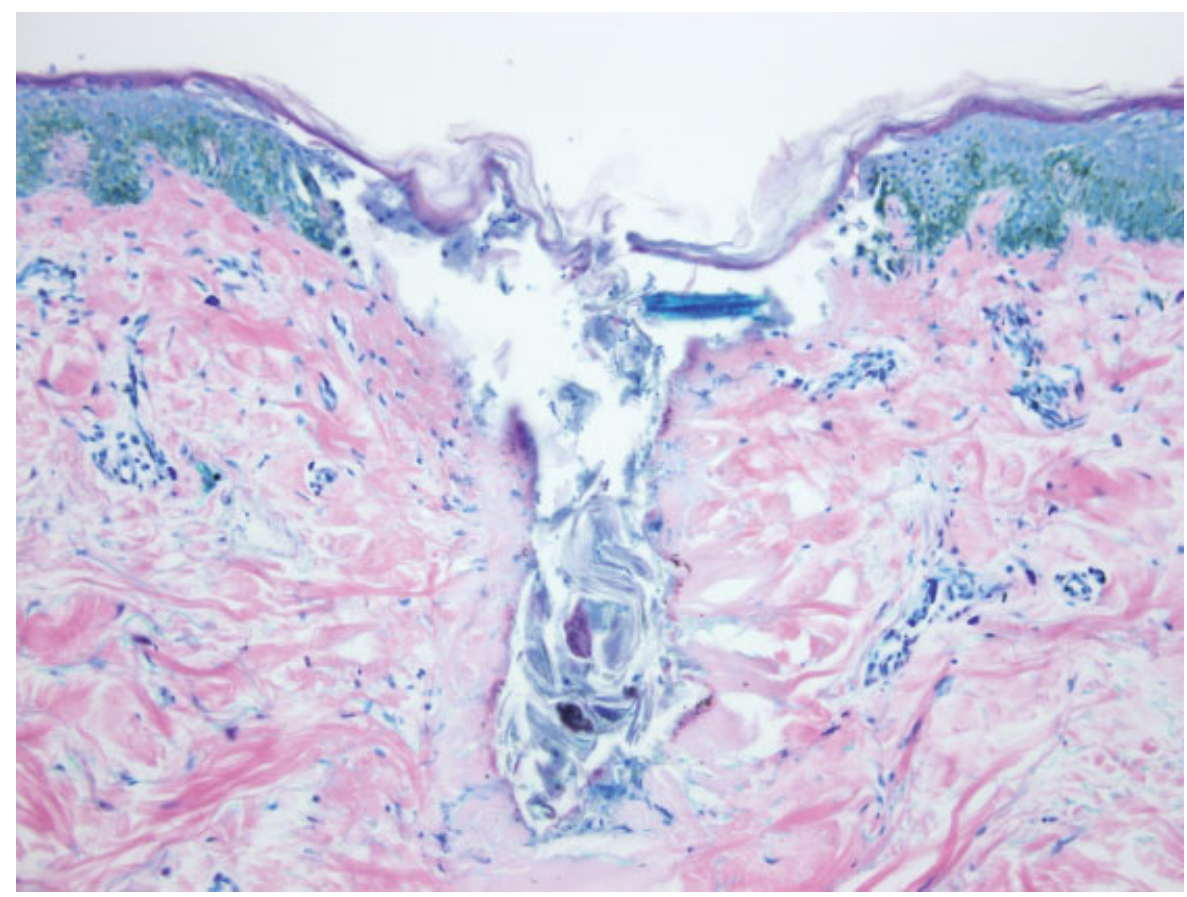

Fig. 3 Hyaluronic acid incorporated post-ablative fractional laser (AFXL) by sonophoresis.

possibly even the subepidermal fat compartment may be addressed.

\section{Intensified PDT to Treat Non-Melanoma Skin Cancer}

It is very promising that the AFXL-intensified PDT has led to the observation of a reduced appearance of developing AK in comparison to PDT standard regimen during 3-month followup. This might be based on improved penetration and distribution of the photosensitizer post-AFXL. ${ }^{18}$ Most probably, this will be the first treatment regimen using the option of a facilitated drug delivery through an artificial TOR of the epidermal barrier. In addition, a first case report shows that even small solid basal cell carcinomas can be treated effectively. ${ }^{21}$ It seems that unifying molecular mechanism of both AFXL and PDT results in synergistic clinical responses (-Fig. 2). ${ }^{22-24}$

\section{Removal of Foreign Bodies Such as Tattoo Ink and Filler}

The transient epidermal opening paired with active MEND expulsion removal of foreign bodies such as tattoo inks, filler substances, or other foreign matter shall be an indication for fractional laser. In case of filling substances, calcium-hydroxylapatite or silicon, clinical efficacy has been demonstrated already. ${ }^{25,26}$ Multicolored tattoos do not respond well to

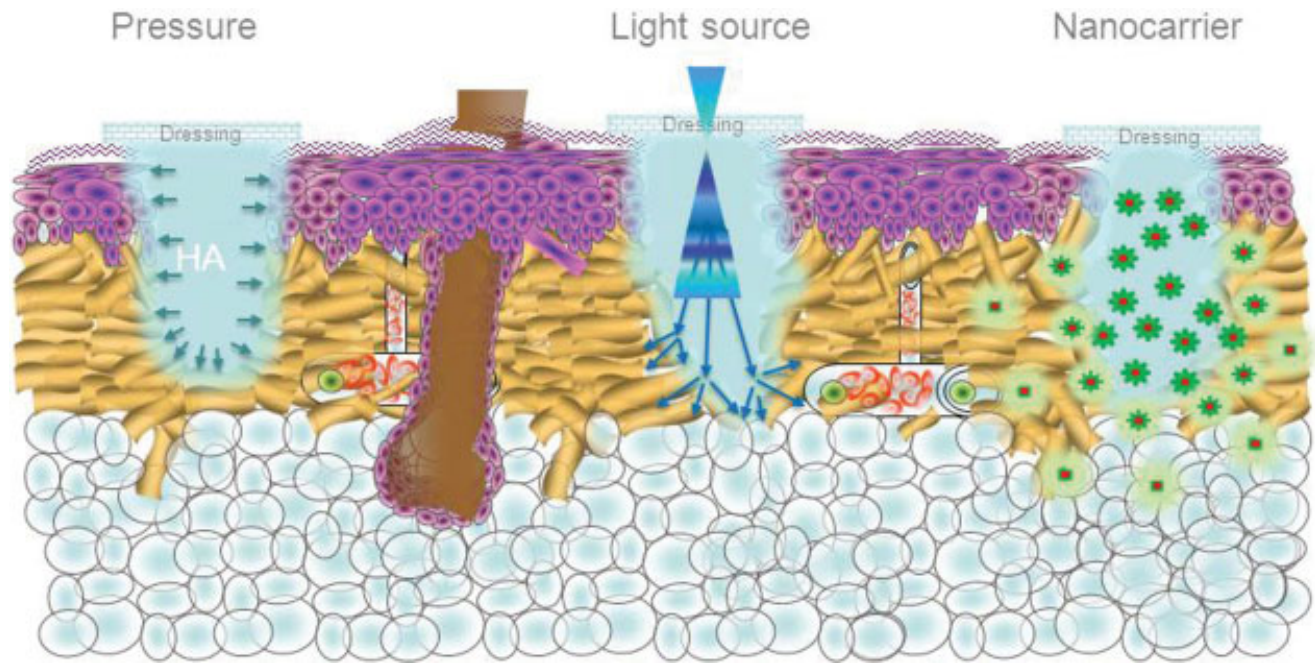

Fig. 4 The optical window. 
conventional q-switched lasers and are indications of allergic reactions. ${ }^{11,27}$ So far, the removal of ink particles has been demonstrated for both $\mathrm{AFXL}^{28}$ and NAFXL ${ }^{29}$ procedures. It would be more than interesting if this concept could be extended to other granuloma, fungi, or parasites. Lately, fractional ablative laser has been reported for the treatment of lichen amyloidosis. ${ }^{30}$

\section{Fibrosing and Inflammatory Skin Diseases}

Skin diseases characterized by chronic inflammation and ongoing fibrosis are difficult to treat. A recent case report demonstrated the efficacy of AFXL in a morphea-related joint contracture, a common complication of linear or generalized morphea. ${ }^{31}$ Although the mechanisms of this kind of remodeling are not fully understood, it sheds light on the potential of fractional laser to be used in those conditions. In fact, during treatments of acne scar, we experienced that inflammatory lesion did also respond to the treatment. ${ }^{32}$ In addition, in a clinical case of pyoderma gangrenosum, the laser treatments did help improve the condition. ${ }^{33}$

\section{Dermal Light Therapy}

Once the TOR has been applied, light may also penetrate to the deeper parts of the skin. Hence, the MAZs are small and light may be scattered at the entrance already and conduits might be kept open for a period of time by filling the holes with optical translucent material. Hyaluronic acid forced by sonophoresis into the MAZ may come into play to fulfill this task (-Fig. 3).

There are potential applications for dermal UV irradiation; for example, treatment of vascular structures by yellow light.

\section{Conclusion}

The new insights of fractional laser treatments have broadened our understanding in post-fractional wound healing. As a consequence, those interventions have become standard applications. On the basis of available published data, recommendations have been made to treat chronic UV damage, wrinkles, and scars.

However, the potential concept has not been used so far. Newer reports describe its efficacy in fibrosing diseases, inflammation, foreign body removal, and other conditions.

The biggest progress arose by using this technique in conjunction with drug delivery. It is very promising that the AFXL-intensified PDT has led to the observation of a reduced appearance of developing AK in comparison to PDT standard regimen during 3-month follow-up. The temporary opening of the epidermal barrier is a new gate to the dermal compartment which enables us to develop new treatment strategies.

To date, AFXL systems are central to laser-assisted drug delivery; hence, it enhances the uptake of topical therapeutics. Superior clinical outcome to treat field cancerization will make the use of this concept worldwide soon. Therefore, all aspects of safety and efficacy have to be addressed first before using it. Long-term data from clinical trials are needed for routine treatments. The fractional laser-assisted drug delivery technique is not FDA approved and, so far, constitutes a research-based off-label indication.

Conflict of Interest

The author has no conflict of interest regarding this publication.

\section{References}

1 Manstein D, Herron GS, Sink RK, Tanner H, Anderson RR. Fractional photothermolysis: a new concept for cutaneous remodeling using microscopic patterns of thermal injury. Lasers Surg Med 2004; 34(5):426-438

2 Paasch U, Haedersdal M. Laser systems for ablative fractional resurfacing. Expert Rev Med Devices 2011;8(1):67-83

3 Grunewald S, Bodendorf M, Illes M, Kendler M, Simon JC, Paasch U. In vivo wound healing and dermal matrix remodelling in response to fractional $\mathrm{CO}(2)$ laser intervention: clinicopathological correlation in non-facial skin. Int J Hyperthermia 2011; 27(8):811-818

4 Polder KD, Harrison A, Eubanks LE, Bruce S. 1,927-nm fractional thulium fiber laser for the treatment of nonfacial photodamage: a pilot study. Dermatol Surg 2011;37(3):342-348

5 Skovbølling Haak C, Illes M, Paasch U, Hædersdal M. Histological evaluation of vertical laser channels from ablative fractional resurfacing: an ex vivo pig skin model. Lasers Med Sci 2011; 26(4):465-471

6 Paasch U, Bodendorf MO, Grunewald S. Dermatologische Lasertherapie: Fraktionale Laser. Berlin: KVM Verlag; 2011

7 Brauer JA, Geronemus RG. Laser treatment in the management of infantile hemangiomas and capillary vascular malformations. Tech Vasc Interv Radiol 2013;16(1):51-54

8 Tsilika K, Bahadoran P, Passeron T. Superficial lymphangioma treated with fractional ablative laser: a case report with clinical and reflectance confocal microscopy evaluation. Dermatol Surg 2013;39(1, Pt 1):141-143

9 Weiss ET, Brauer JA, Anolik R, et al. 1927-nm fractional resurfacing of facial actinic keratoses: a promising new therapeutic option. J Am Acad Dermatol 2013;68(1):98-102

10 Helsing P, Togsverd-Bo K, Veierød MB, Mørk G, Haedersdal M. Intensified fractional $\mathrm{CO} 2$ laser-assisted photodynamic therapy vs. laser alone for organ transplant recipients with multiple actinic keratoses and wart-like lesions: a randomized half-side comparative trial on dorsal hands. Br J Dermatol 2013;169(5): 1087-1092

11 Ibrahimi OA, Syed Z, Sakamoto FH, Avram MM, Anderson RR. Treatment of tattoo allergy with ablative fractional resurfacing: a novel paradigm for tattoo removal. J Am Acad Dermatol 2011; 64(6):1111-1114

12 Baker DJ, Wijshake T, Tchkonia T, et al. Clearance of p16Ink4apositive senescent cells delays ageing-associated disorders. Nature 2011;479(7372):232-236

13 Haedersdal M, Sakamoto FH, Farinelli WA, Doukas AG, Tam J, Anderson RR. Fractional $\mathrm{CO}(2)$ laser-assisted drug delivery. Lasers Surg Med 2010;42(2):113-122

14 Haedersdal M, Katsnelson J, Sakamoto FH, et al. Enhanced uptake and photoactivation of topical methyl aminolevulinate after fractional CO2 laser pretreatment. Lasers Surg Med 2011;43(8): 804-813

15 Lee WR, Shen SC, Al-Suwayeh SA, Yang HH, Yuan CY, Fang JY. Laserassisted topical drug delivery by using a low-fluence fractional laser: imiquimod and macromolecules. J Control Release 2011; 153(3):240-248 
16 Beltraminelli H, Dietrich N, Hunziker T. Fractional transepidermal delivery: a histological analysis. Dermatology 2011;223(4): 321-324

17 Togsverd-Bo K, Lerche CM, Philipsen PA, Poulsen T, Wulf HC, Haedersdal M. Porphyrin biodistribution in UV-exposed murine skin after methyl- and hexyl-aminolevulinate incubation. Exp Dermatol 2012;21(4):260-264

18 Togsverd-Bo K, Haak CS, Thaysen-Petersen D, Wulf HC, Anderson RR, Hædersdal M. Intensified photodynamic therapy of actinic keratoses with fractional $\mathrm{CO} 2$ laser: a randomized clinical trial. $\mathrm{Br} \mathrm{J}$ Dermatol 2012;166(6):1262-1269

19 Wu X, Landfester K, Musyanovych A, Guy RH. Disposition of charged nanoparticles after their topical application to the skin. Skin Pharmacol Physiol 2010;23(3):117-123

20 Peer D, Karp JM, Hong S, Farokhzad OC, Margalit R, Langer R. Nanocarriers as an emerging platform for cancer therapy. Nat Nanotechnol 2007;2(12):751-760

21 Haedersdal M, Togsverd-Bo K, Paasch U. Case reports on the potential of fractional laser-assisted photodynamic therapy for basal cell carcinomas. Lasers Med Sci 2012;27(5):1091-1093

22 Helbig D, Simon JC, Paasch U. Photodynamic therapy and the role of heat shock protein 70. Int J Hyperthermia 2011;27(8):802-810

23 Helbig D, Paasch U. Molecular changes during skin aging and wound healing after fractional ablative photothermolysis. Skin Res Technol 2011;17(1):119-128

24 Helbig D, Moebius A, Simon JC, Paasch U. Nonablative skin rejuvenation devices and the role of heat shock protein 70 : results of a human skin explant model. J Biomed Opt 2010;15(3):038002

25 Reddy KK, Brauer JA, Anolik R, et al. Calcium hydroxylapatite nodule resolution after fractional carbon dioxide laser therapy. Arch Dermatol 2012;148(5):634-636
26 Cho S, Jung JY, Shin JU, Lee JH. Silicone-induced foreign body reaction of the face successfully treated using nonablative 1,550 nm erbium-glass and ablative 10,600 -nm carbon dioxide fractional lasers. Dermatol Surg 2012;38(10):1744-1746

27 Weiss ET, Geronemus RG. Combining fractional resurfacing and Qswitched ruby laser for tattoo removal. Dermatol Surg 2011;37(1): 97-99

28 Wang CC, Huang CL, Sue YM, Lee SC, Leu FJ. Treatment of cosmetic tattoos using carbon dioxide ablative fractional resurfacing in an animal model: a novel method confirmed histopathologically. Dermatol Surg 2013;39(4):571-577

29 Wang CC, Huang CL, Lee SC, Sue YM, Leu FJ. Treatment of cosmetic tattoos with nonablative fractional laser in an animal model: a novel method with histopathologic evidence. Lasers Surg Med 2013;45(2):116-122

30 Anitha B, Mysore V. Lichen amyloidosis: novel treatment with fractional ablative 2,940 nm Erbium: YAG laser treatment. J Cutan Aesthet Surg 2012;5(2):141-143

31 Kineston D, Kwan JM, Uebelhoer NS, Shumaker PR. Use of a fractional ablative 10.6- $\mu \mathrm{m}$ carbon dioxide laser in the treatment of a morphea-related contracture. Arch Dermatol 2011;147(10): $1148-1150$

32 Isarría MJ, Cornejo P, Muñoz E, Royo de la Torre J, Moraga JM. Evaluation of clinical improvement in acne scars and active acne in patients treated with the $1540-\mathrm{nm}$ non-ablative fractional laser. J Drugs Dermatol 2011;10(8):907-912

33 Park J, Choi MJ, Goo B, Cho SB. Refractory pyoderma gangrenosum effectively treated using a $10,600-\mathrm{nm}$ carbon dioxide fractional laser. Dermatol Surg 2013;39(3, Pt 1):477-479

34 Paasch U, Bodendorf MO, Grunewald S. Dermatologische Lasertherapie: Fraktionale Laser. Berlin: KVM Verlag; 2012 